MEDICAL RESEARCH

\section{On the trail of invasive cells in breast cancer}

\section{During breast-cancer progression, tumour cells that arise in the milk duct spread elsewhere in the breast. The origin of these invasive tumour cells is now revealed by an analysis of spatially defined single cells.}

\section{DOUGLAS S. MICALIZZI}

\section{\& SHYAMALA MAHESWARAN}

$\mathrm{I}$ $\mathrm{n}$ the early-stage breast cancer called ductal carcinoma in situ (DCIS), a cluster of cancer cells arises in the milk duct and remains confined there. The recorded incidence of DCIS has risen since the late 1980s, probably due to an increase in its detection through more widespread breast-cancer screening ${ }^{1}$. Up to $40 \%$ of cases of DCIS progress to invasive ductal carcinoma (IDC), in which tumour cells invade other regions of the breast ${ }^{2-4}$. IDC requires clinical treatment. Moreover, because it is not possible to predict which people with DCIS are at risk of progressing to IDC, the standard treatment for patients who have DCIS is surgery, often followed by radiotherapy ${ }^{5}$. Therefore, understanding how DCIS progresses to IDC might enable the selective treatment of those at high risk of developing IDC. A paper in Cell by Casasent et al. ${ }^{6}$ reveals the origin of DCIS and its progression to IDC.

Casasent and colleagues obtained frozen samples of breast-cancer tissue gathered from patients. The authors used single-cell DNA sequencing of cells from DCIS and IDC regions in the same section of tissue to create molecular maps of patients' breast cancers, in an approach they call topographic single-cell sequencing. They stained the samples to identify tumour cells and noted the location of each selected cell in the regions of DCIS and IDC. A technique called laser capture microdissection enabled the separation of a selected cell from its neighbours by tracing a laser beam around the cell. This was combined with an approach termed laser catapulting, in which energy from an ultraviolet laser propels a microdissected cell into a collection tube for subsequent DNA analysis. These laser-based techniques are used in other contexts, including forensic science and studies of plant physiology?

The authors analysed 1,293 of these individually isolated tumour cells from 10 patients with breast cancer. They assessed whether the cells isolated from each patient contained alterations in the number of copies of genes, a type of change that sometimes drives tumour formation and growth. A comparison of these alterations in tumour cells from an individual can identify distinct populations of cells (clones). Analysis of the patterns of genetic alterations can reveal a clone's cellular origin and its relationship with other clones. Combining this information with knowledge of a cell's location offers a way to track the progression of DCIS to IDC.

Casasent et al. observed that the tumours of four patients had formed from a single clone, and that those of the other six patients consisted of between 2 and 5 clones. The clones of each patient with multiclonal tumours shared a set of genomic aberrations, which suggests that all tumour cells in each of those patients had descended from a single cell, with genetic divergence between the various clones arising from the acquisition of further alterations.

In patients with multiclonal tumours, all of the individual's clones were present in both DCIS and IDC regions (Fig. 1). This points to a model in which clones arise and evolve in the milk duct and then escape to establish IDC elsewhere in the breast, such as in regions containing stromal cells. The various clones had a wide distribution across breast tissue, with certain clones being evenly distributed between milk ducts and regions of tumour invasion, and others being enriched in either the invaded regions or the milk ducts.

The authors conducted additional analysis by sequencing the protein-coding regions of the tumour cells' DNA - detecting an average of 23 mutations per patient. This analysis confirmed that there were striking similarities between specific clones in a given patient, regardless of whether the clonal tumour cell was part of a DCIS or an IDC region. The transition from DCIS to IDC was not associated with a notable increase in the number of mutations in the clones or in the number of independent clones. A few mutations specific to DCIS or IDC regions were identified, and, although these warrant further study, the authors' main finding is the remarkable genetic similarity of a patient's tumour cells in these two distinct disease states. The observation that genomic evolution of tumour cells occurs in the milk duct before invasion proceeds suggests that a transient period of genome instability is an early event in breast-cancer initiation.

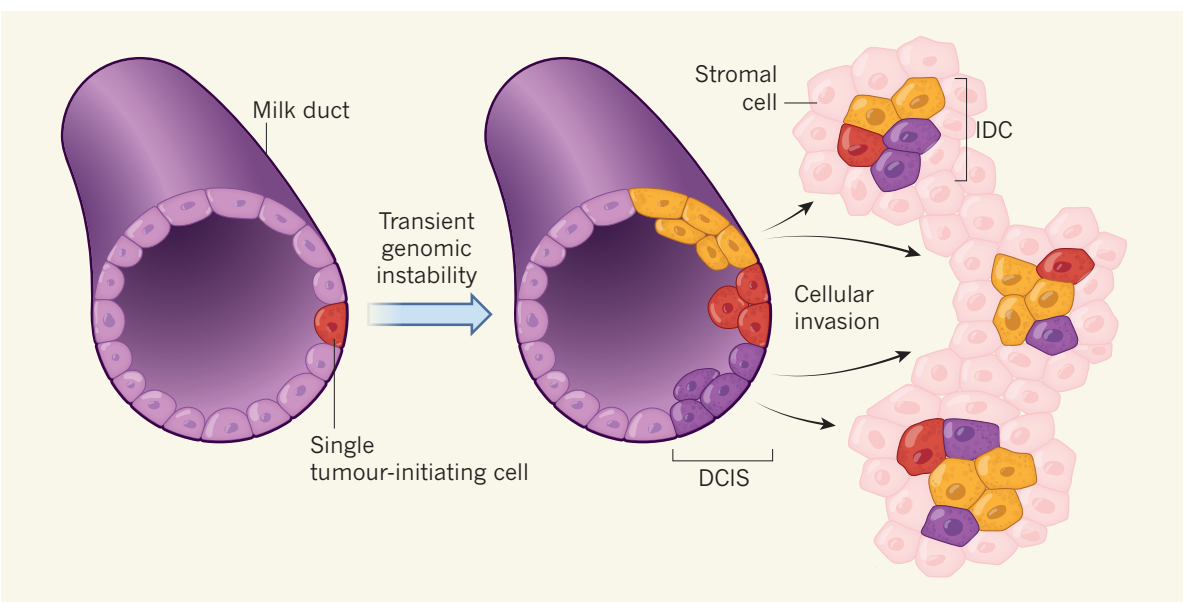

Figure 1 | Tracking the cellular origins of breast cancer. Casasent et al. ${ }^{6}$ report the development of a technique for spatially resolved analysis of individual breast-cancer cells in tissue samples obtained from patients. This enabled them to determine the cellular origin of a non-invasive breast cancer called ductal carcinoma in situ (DCIS), in which tumour cells form in the milk duct. The authors reveal that DCIS arises from a single tumour-initiating cell and its descendants. Some of these tumour cells might diverge from each other during a period of transient genomic instability to form several different cellular populations, or clones (distinct clones shown as red, yellow or dark purple). DCIS sometimes progresses to an invasive stage in which tumour cells migrate to other areas of the breast tissue, such as regions containing stromal cells, where tumour growths known as invasive ductal carcinoma (IDC) form. The identity of the cells that give rise to IDC has been debated. Casasent and colleagues found that all the tumour clones present in regions of DCIS are also present in areas of IDC. 
Casasent and colleagues' model, in which breast cancer arises in the milk duct from a single initiating cell that gives rise to several clones in the duct that then invade the rest of the breast tissue, contrasts with two alternative models of IDC development ${ }^{8-10}$. The independent-lineage model $^{8,9}$ proposes that DCIS and IDC evolve independently from different tumour-initiating cells. The other model ${ }^{10}$, called an evolutionary bottleneck, proposes that a single initiating cell in the milk duct gives rise to several clones, only one of which successfully escapes the milk duct to invade the rest of the tissue.

Many questions remain. What triggers the genetic alterations in the tumour-initiating cell in DCIS? Do other potential tumour-initiating cells arise at the earliest stage of the disease, of which only one gives rise to DCIS and the others are eliminated? A better understanding is needed of how tumour cells escape the milk duct through the breakdown of the duct's outer layer (the basement membrane), and how some cells from all the clones present in DCIS manage to exit the duct.
The genetic similarity between tumour cells in the DCIS and IDC regions of a given individual raises the question of what differences enable some cells to give rise to invasive disease. A clearer definition of the differences between low-risk DCIS that does not advance to IDC and high-risk DCIS that does, might enable the accurate prediction of disease-progression risk. Addressing this will probably require single-cell comparisons of gene-transcription profiles or the assessment of modifications known as epigenetic changes, which alter the protein-DNA complex of chromatin and can affect gene expression.

Casasent and colleagues' approach could be used to study different cancers in which spatially resolved insight into tumour growth cannot be obtained by conventional methods. For example, using this technique to investigate abnormal cellular growths that eventually progress to a malignant state would be a good choice for future studies into the origin and clonal heterogeneity of tumour cells. It is unclear how widely this method is likely to be applied, considering the technical and financial requirements. Nevertheless, Casasent et al. have demonstrated that their approach has the potential to provide useful insight into the dynamics of tumour clones during the progression of breast cancer.

\section{Douglas S. Micalizzi and Shyamala}

Maheswaran are at Massachusetts General Hospital Center for Cancer Research, Harvard Medical School, Massachusetts 02129, USA. e-mail:maheswaran@helix.mgh.harvard.edu

1. Virnig, B. A., Tuttle, T. M., Shamliyan, T. \& Kane, R. L. J. Natl Cancer Inst. 102, 170-178 (2010).

2. Sanders, M. E., Schuyler, P. A., Dupont, W. D. \& Page, D. L. Cancer 103, 2481-2484 (2005).

3. Sanders, M. E., Schuyler, P. A. Simpson, J. F., Page, D. L. \& Dupont, W. D. Mod. Pathol. 28, 662-669 (2015).

4. Collins, L. C. et al. Cancer 103, 1778-1784 (2005).

5. Shah, C. et al. JAMA Oncol. 2, 1083-1088 (2016).

6. Casasent, A. K. et al. Cell 172, 205-217 (2018).

7. Schütze, K., Niyaz, Y., Stich, M. \& Buchstaller, A. Methods Cell Biol. 82, 649-673 (2007)

8. Sontag, L. \& Axelrod, D. E. J. Theor. Biol. 232, 179-189 (2005).

9. Miron, A. et al. Cancer Res. 70, 5674-5678 (2010). 10.Cowell, C. F. et al. Mol. Oncol. 7, 859-869 (2013). 